Introduction: In June 2012, the federal government made cuts to the Interim Federal Health (IFH) Program that reduced or eliminated health insurance for refugee claimants in Canada. The purpose of this study was to examine the effect of the cuts on emergency department (ED) use among patients claiming IFH benefits. Methods: We conducted a health records review at two tertiary care EDs in Ottawa. We reviewed all ED visits wherein an IFH claim was made at triage, for 18 months before and 18 months after the changes to the program on June 30, 2012 (20112013). Claims made before and after the cuts were compared in terms of basic demographics, chief presenting complaints, acuity, diagnosis, presence of primary care, and financial status of the claim. Results: There were a total of 612 IFH claims made in the ED from 2011-2013. The demographic characteristics, acuity of presentation and discharge diagnosis were similar during both the before and after periods. Overall, $28.6 \%$ fewer claims were made under the IFH program after the cuts. Of the claims made, significantly more were rejected after the cuts than before $(13.7 \%$ after vs. $3.9 \%$ before, $\mathrm{p}<0.05)$. The majority $(75.0 \%)$ of rejected claims have not been paid by patients. Fewer patients after the cuts indicated that they had a family physician $(20.4 \%$ after vs. $30 \%$ before, $\mathrm{p}<0.05$ ) yet a higher proportion of these patients were still advised to follow up with their family doctor during the after period (67.2\% after vs. $41.8 \%$ before, $\mathrm{p}<0.05$ ). Conclusion: A higher proportion of both rejected and subsequently unpaid claims after the IFH cuts in June 2012 represents a potential barrier to emergency medical care, as well as a new financial burden to be shouldered by patients and hospitals. A reduction in IFH claims in the ED and a reduction in the number of patients with access to a family physician also suggests inadequate care for this population. Yet, the lack of primary care was not reflected in the follow-up advice offered by ED physicians to patients. Keywords: refugee, Interim Federal Health (IFH), international

\section{P012 \\ Equity of care between First Nations and non-First Nations patients in Saskatoon emergency departments}

R. Batta, BSc, J. Stempien, BSc, MD, M.A. Sasbrink-Harkema, BSc, MD, T.O. Oyedokun, MBChB, R. Carey, BSc; University of Saskatchewan, Saskatoon, SK

Introduction: Studies have shown that First Nations patients have poorer health outcomes than non-First Nations patients. This has raised concerns that they receive unequal treatment from the health care system in general and the Emergency Department (ED) in particular. We sought to determine if there was such a difference and what it was so that it could be corrected. Methods: We performed a retrospective chart review to compare the care received by status First Nations and non-First Nations patients presenting to two hospital ED's (Royal University Hospital and St. Paul's Hospital) in Saskatoon, Saskatchewan with the chief complaint of abdominal pain and a Canadian Triage and Acuity Scale (CTAS) score of three. A total of 200 charts were reviewed (100 from each site and 100 from each group) by two medical students. One student was involved in blinding the charts; the other was responsible for analyzing the charts. Identifying information on the charts was redacted to blind the reviewer to the patient's group during the chart review. Data extracted from each chart included time to doctor, time to analgesia given, length of stay, referral for consultation, blood work, imaging, bounce backs, reassessment, physical and history exam, and final disposition. This data will then be compared between the two population groups to find if there is equality in care given. Results: Data is currently being analyzed and will be available for presentation at
CAEP 2016. Conclusion: The goal of our health care system is to provide the same level of excellent care to every patient that arrives in the ED. If care is not being provided equitably to First Nations patients this must be identified for it to be addressed. This study aimed to determine whether disparities in care exist. If they are found subsequent research could be done to determine why these differences exist while at the same time working to minimize and eliminate them for the benefit of First Nations patients.

Keywords: triage, First Nations, equity

\section{P013}

Inter-facility transfers for CT scans from a rural emergency department: a pilot study

C. Bergeron, I. Lavallée-Bourget, F.K. Tounkara, R. Fleet, MD, PhD; Université Laval, Québec City, QC

Introduction: Rural emergency departments (EDs) are an important gateway to care for the $20 \%$ of Canadians who live in rural areas. We recently reported that fewer than $15 \%$ of rural EDs in Canada have access to a CT scanner. Lack of CT scanners in rural hospitals can result in frequent inter-facility transfers and delays in diagnosing and treating life-threatening conditions. No recent study has examined this issue. Objective: With a future larger study in mind, we did a pilot assessment of inter-facility transfers for CT scans from one rural ED and evaluated the quality of the data and feasibility. Methods: This pilot study was part of our province-wide study on rural emergency care. Criteria were having 24/7 physician coverage and acute-care hospitalization beds. The hospital was also selected for its proximity and local interest. Two medical students collected data from hospital databases to determine annual number of ED visits, ED transfers, proportion of transfers for CT scans, reasons for examinations, and transfer times from April 1, 2010 to March 31, 2015. Descriptive statistics were reported as well as data quality and feasibility indicators. Results: For each year from 2010 to 2014 , there was an average of 13,341 ED visits, 444 inter-facility transfers, and $125 \mathrm{CT}$ scans. Over the five years an average of $28 \%$ of the inter-facility transfers were for CT scans, and the majority were abdominal CT scans. Inter-facility transfer data was $100 \%$ accessible through hospital databases but inter-facility transfer times and final diagnoses were not. Conclusion: More than a quarter of inter-facility transfers were for CT imaging. The limited electronic data in this Quebec rural ED precluded analysis of inter-facility times. While further cost-benefit analysis is required, preliminary data suggests local CTs may save time, money and lives.

Keywords: rural emergency departments, computed tomography, inter-facility transfer

\section{P014}

An investigation to determine if being roomed next to a psychiatric patient affects patient satisfaction and perception of care in those not being evaluated for a psychiatric complaint

F.A. Blais, MD; Vidant Medical Center, Greenville, NC

Introduction: Nearly 12 million emergency department (ED) visits in the USA annually are related to a mental health and/or substance abuse condition. This is equivalent to 1 out of every $8 \mathrm{ED}$ visits or 12.5 percent of all ED visits annually. States cut $\$ 5$ billion in mental health services from 2009 to 2012. In the same period, the country eliminated at least 4,500 public psychiatric hospital beds. This has led to an increase in psychiatric boarding. Boarding consumes scarce ED resources and prolongs the amount of time that all patients must spend waiting for services. The aim of this study is to determine if being 
roomed next to a psychiatric patient affects patient satisfaction and perception of care. Methods: A survey consisting of 15 patient satisfaction questions was distributed to patients over a period of three months in the ED at a tertiary care center with $>125,000$ visits a year. Patients included were English-speaking adults (18 years or older) with an Emergency Severity Index of 3-5. Responses were analyzed with a chi-square across 2 groups with p-value of 0.05 considered as significant. Results: A convenience sample of 78 surveys was obtained. 40 surveys were completed by those roomed next to a patient with a psychiatric complaint and 38 surveys were completed by patients not roomed next to a patient being seen for a psychiatric complaint. For every satisfaction question asked, the patients placed away from mental health encounters gave significantly higher ratings than the patients roomed near psychiatric patients. Patients roomed next to psychiatric patients had a statistically significant decrease in satisfaction in nursing attentiveness, nursing promptness in responding to the call bell, attentiveness of the physician team, and of the overall encounter itself. All values were significant with all but one $p$-value being $<0.01$. There was no difference between the 2 groups with respect to gender, age range, reason for visit or wait time. Conclusion: This study suggests that patients being roomed next to a patient with a psychiatric complaint had significantly decreased patient satisfaction.

Keywords: patient satisfaction, psychiatric complaint

\section{P015}

Implementing the Canadian CT Head Rule in a community emergency department

G. Bock, MD, R. Setrak, MD, S. Freeman, BSc; McMaster University Niagara, St. Catharines, ON

Introduction: The Canadian CT Head Rule ('the rule') is widely used across the country and its use is specifically recommended by Choosing Wisely Canada. Studies in Canadian hospitals have shown appropriate declines in CT scans when decision tools have been made readily available and useable at the point of care. Research into the implementation of the Canadian CT Head Rule in particular has shown that barriers to its use include an inability to accurately recall each criteria and forgetting to attempt to apply the rule altogether. In an attempt to provide our clinicians with effective access to the rule, we modified CT requisitions and order procedures to facilitate the use of the rule for every head CT in our emergency department (ED). Methods: A quality improvement (QI) approach was used to pilot, implement, and evaluate the modified CT requisition at our hospital. Several Plan-Do-Study-Act cycles involving stakeholders in the hospital resulted in iterative changes to the requisition leading to the implemented version. The new requisition required physicians to indicate which rules or exclusion criteria were met and this was made mandatory for all head CTs ordered. Demographic data was collected on all patients presenting to the ED on age, gender, CTAS level, disposition, and length of stay. Data on which exclusion criteria were appropriate, the rules met leading to CT scans, whether each requisition was used appropriately, and whether there was a significant injury found was collected for each patient receiving a head CT after implementation. Results: In our primary outcome (\% of ED visits receiving a head $\mathrm{CT}$ ), preliminary results have demonstrated a relative reduction in head CT ordering of $10.9 \%$. Our study at completion is powered to detect a $\sim 10 \%$ relative change in ordering behaviour, and a Chi square of the data to date yields a P-value of 0.0147 . There are no significant differences in visit volume or any of the demographics collected to date. Final results including analysis are anticipated in March, 2016. Conclusion: Preliminary results on this simple, no-cost intervention are very promising. The reduction in head
CTs ordered suggests that with mandated access to an easy-to-use, well validated decision tool, ED physicians have been able to confidently defer scans that have a very low risk of having any significant injury present, reducing cost, radiation exposure, and perhaps time in department.

Keywords: decision tool, computed tomography, quality improvement

\section{P016}

Evaluating the impact of a novel mobile care team (MCT) on the prevalence of ambulatory care sensitive conditions presenting to emergency medical services in Nova Scotia

D.R. Brown, MPH, A. Carter, MD, J. Goldstein, PhD, J. Jensen, MAHSR, A. Travers, MD, MSc, T. Dobson, MPH, P. Cosgrove; Emergency Health Services, Sydney, NS

Introduction: Hospitalization due to ambulatory care sensitive conditions (ACSC) is a proxy measure for access to primary care. Emergency medical services (EMS) are increasingly called when primary care cannot be accessed. A novel paramedic-nurse EMS Mobile Care Team (MCT) was implemented in an under-serviced community. The MCT responds in a non-transport unit to bookings from EMS, emergency and primary care and to low-acuity 911 calls in a defined geographic region. Our objective was to compare the prevalence of ACSC in ground ambulance (GA) responses before and after the introduction of the MCT. Methods: A cross-sectional analysis of GA and MCT patients with ACSC (determined by chief complaint, clinical impression, treatment protocol and medical history) one year pre- and one year post-MCT implementation was conducted for the period Oct. 1, 2012 to Sept. 30, 2014. Demographics were described. Predictors of ACSC were identified via logistic regression. Prevalence was compared with chi-squared analysis. Results: There were 975 calls pre- and 1208 GA/95 MCT calls post-MCT. ACSC in GA patients preand post-MCT was similar: $\mathrm{n}=122,12.5 \%$ vs. $\mathrm{n}=185,15.3 \%$; $\mathrm{p}=0.06$. ACSC in patients seen by EMS (GA plus MCT) increased in the post-period: $122(12.5 \%)$ vs. $204(15.7 \%) \mathrm{p}=0.04$. Pre vs post, GA calls differed by sex $(\mathrm{p}=0.007)$ but not age $(65.38 \pm 15.12$ vs. $62.51 \pm 20.48 ; \mathrm{p}=0.16$ ). Post-MCT, prevalence of specific ACSC increased for GA: hypertension $(\mathrm{p}<0.001)$ and congestive heart failure $(\mathrm{p}=0.04)$. MCT patients with ACSC were less likely to have a primary care provider compared to GA $(90.2 \%$ and $87.6 \%$ vs. $63.2 \% ; p=0.003$, $\mathrm{p}=0.004)$. Conclusion: The prevalence of ACSC did not decrease for GA with the introduction of the MCT, but ACSC in the overall patient population served by EMS increased. It is possible more patients with ACSC call or are referred to EMS for the new MCT service. Given that MCT patients were less likely to have a primary care provider this may represent an increase in access to care, or a shift away from other emergency/episodic care. These associations must be further studied to inform the ideal utility of adding such services to EMS and healthcare systems.

Keywords: emergency medical services (EMS), ambulatory care sensitive conditions, community paramedicine

\section{P017}

Does a busy day predict another busy day? A time-series analysis of multi-centre emergency department volumes

M.B. Butler, MSc, H. Gu, PhD, T. Kenney, PhD, S.G. Campbell, MB, BCh; Dalhousie University, Halifax, NS

Introduction: Variations of patient volumes in the ED according to days of the week and month of the year are well-established. Anecdotally, ED volumes follow 'waves' that correlate with previous 